management and psychological care to enable weaning from ventilation and a comfortable death.

Conclusion This new and unique role has reinforced the value of Consultant nursing practice at a clinical level, influencing and shaping nursing care in a live way. The transferable skills held have enabled dynamic nursing that could be seen as a blueprint for promoting a workforce development that is more resilient for the future, valuing the differing perspectives of nursing practice in both primary and secondary care.

\section{P-221 A TALE OF TWO HOSPICES: THE DEVELOPMENT OF A UNIQUE SERVICE MODEL OF HOSPICE CONSULTANT SUPPORT}

${ }^{1}$ Eilidh Dear, ${ }^{1}$ Jill McKane, ${ }^{2}$ Amy Proffitt, ${ }^{1}$ Paul Coulter, ${ }^{1}$ Linda McEnhill, ${ }^{2}$ Robert George. ${ }^{1}$ Ardgowan Hospice, Greenock, UK; ${ }^{2}$ St Christopher's Hospice, London, UK

10.1136/spcare-2021-Hospice.236

Background The Association of Palliative Medicine of Great Britain and Ireland (APM) state 'Palliative medicine is at a turning point in terms of delivering new service models' (Workforce Committee of the APM, 2019).

Ardgowan Hospice has had consultant recruitment challenges. Explanations include geography and no Clyde palliative trainee placements. There was an estimated 56 vacant UK consultant posts in 2018. Too few trainees and the removal of the CESR process means the number of prospective consultants is insufficient for service demand. A changing teaching curriculum and seven-day working creates concerns for junior-tier rotas which will impact the consultant on-call. With an ever-increasing demand of services, innovative staffing solutions are required so palliative medicine can continue to flourish in hospitals, hospices, and community.

Ardgowan Hospice (AH) and St Christopher's Hospice $(\mathrm{SCH})$ developed an innovative service model for hospice consultant support.

Aim To develop and evaluate a remote consultant service model.

Method Quality improvement methodology enabled the design, development, and evaluation of remote consultant framework. Plan Do Study Act (PDSA) cycles, cause and effect analysis, field analysis, survey, and audit were all utilised.

Results

- In 2019, PDSA cycles showed that it was imperative that shared policies, guidelines, and protocols were established to provide seamless patient care.

- Quality improvement analysis highlighted important themes for cohesive working. These were team-work, flexibility and adaptability, continuity of care, as well as high quality handovers.

- Innovative IT solutions were developed to provide efficient communication within clinical governance structures.

- A 2021 audit showed 11 communications with SCH about 12 patients over a three-months period. This facilitated appropriate senior advice when required.

- A 2021 survey was conducted. The average satisfaction score was $8.5 / 10$. A number of qualitative themes were identified.

Conclusion $\mathrm{AH}$ and $\mathrm{SCH}$ have shown that by completing a quality improvement project that it is possible to create an innovative remote consultant service provision model.

\section{P-222 DESIGNING A MOBILE APP WHICH SUPPORTS UNPAID CARERS TO ADMINISTER SUBCUTANEOUS INJECTIONS}

${ }^{1}$ Claudia Hopkins, ${ }^{2}$ Imogen Eastwood, ${ }^{3}$ Marlise Poolman, ${ }^{1}$ Ivor Williams, 'Lily Hoskin, ${ }^{1}$ Cat Kilkenny, ${ }^{1}$ Jonathan Gregory. ${ }^{1}$ Helix Centre, Imperial College, London, UK; ${ }^{2}$ Central North West London NHS Trust, London, UK; ${ }^{3}$ Bangor University, Bangor, UK

\subsection{6/spcare-2021-Hospice.237}

In the UK, most people wish to be at home when they die. Unpaid carers (family and friends) play a key role in ensuring a home death and many are willing to be trained to administer as-needed subcutaneous injections for breakthrough symptoms, to ensure timely symptom control for the person they are caring for. A team in North Wales developed the CARiAD package to train and support such carers, a new practice to most of the UK.

It is hypothesised that a digital app may be beneficial for both carers and the healthcare teams. It could provide more guidance, improve data collection, help facilitate monitoring through the real-time collection of data and support audit data collection. Therefore the Helix Centre, Central North West London (CNWL) NHS Trust and the CARiAD team have collaborated to design and develop a mobile app. We acknowledge that a digital product will not be right for every carer but it is essential to understand whether a digital offering can deliver the intended benefits.

A user-centred design approach was adopted by conducting moderated usability sessions with carers who had previously given subcutaneous as-needed medications. Testing user flows, content and barriers of using paper and digital versions together. After each round, we synthesised the qualitative feedback and made improvements. A pre-pilot study of the app is commencing imminently which will inform a formal pilot study in CNWL of a digital-first offering. If successful a healthcare practitioner view will be developed to aid remote monitoring and support carers.

The design process worked as intended. Unforeseen usability and accessibility issues were identified. Iterative improvement of the app has delivered a product that is well aligned with carers' perspectives and knowledge. We hope this will ensure that the app is easier and safer to use when supporting carers to administer and record as-needed subcutaneous injections.

\section{P-223 SOCIAL WORK LED CARE COORDINATION WITHIN A COMMUNITY PALLIATIVE CARE TEAM}

Catherine Thompson. Kirkwood Hospice, Huddersfield, UK

10.1136/spcare-2021-Hospice.238

The Kirkwood recognised through its user engagement with patients and carers that coordinating palliative health and social care for patients and carers can be extremely complex, confusing and stressful. It appeared to be a contributing factor to carer stress. The crossover from social care to healthcare was often very abrupt with no lead support along the way. It was identified that having a lead service able to remove some of the strain and pressure was needed with the right level of skills and knowledge to support and navigate the system. 